\title{
La Corrección de los Tests de Pensamiento Divergente: Controversias y Soluciones
}

\author{
Scoring Divergent Thinking Tests: Controversies and Solutions
}

\author{
Mercedes Ferrando Prieto ${ }^{1}$, Carmen Ferrándiz García ${ }^{2}$, Marta Sainz Gómez ${ }^{3}$ \\ y María Dolores Prieto Sánchez ${ }^{4}$
}

\begin{abstract}
Resumen
Existen controversias sobre la corrección y puntuación de las pruebas de Pensamiento Divergente, especialmente en Originalidad y Elaboración, planteándose distintas alternativas para puntuarlas. El objetivo es estudiar un procedimiento alternativo para puntuar las dos dimensiones en el Test de Pensamiento Creativo de Torrance (TTCT; Torrance, 1974). Han participado 649 alumnos (5-12 años). El TTCT fue corregido según el manual (puntuaciones sumativas) y calculando la puntuación promedio de Originalidad y Elaboración. Se estudian las implicaciones de este sistema de puntuación: la estructura factorial del test y la identificación del potencial creativo. Se aborda la relación entre Fluidez-Originalidad y Fluidez-Elaboración, tema debatido en la literatura. Los resultados indican que la corrección basada en puntuaciones promedio, afecta a la estructura interna de la prueba, pero no a la identificación del potencial creativo. Los resultados apoyan la idea de que un perfil creativo combina las dimensiones de Fluidez, Originalidad y Elaboración.
\end{abstract}

Palabras clave: evaluación del pensamiento divergente, originalidad, fluidez, elaboración

\begin{abstract}
There are some controversies about the correction and scoring of Divergent Thinking tests, particularly regarding Originality and Elaboration. Thus, different alternatives have been proposed to score them. The aim of this work is to study an alternative procedure to score the two dimensions in the Torrance Creative Thinking Test (TTCT, Torrance, 1974). The sample comprised 649 students (aged between 5-12 years old). The TTCT was corrected according to the manual (summative scores) and calculating the average score of Originality and Elaboration. The implications of this scoring system are analyzed: the factorial structure of the test and the creative potential's identification. Also, the relationship between Fluency-Originality and Fluency-Elaboration are studied. The results indicate that the alternative scoring of the test affects the internal structure, but not the identification of the creative potential. The results support the idea that a highly creative profile combines the dimensions of Fluency, Originality and Elaboration.
\end{abstract}

Keywords: divergent thinking assessment, originality, fluency, elaboration

\footnotetext{
${ }^{1}$ Doctora por la Facultad de Psicología. Profesora Titular de la Universidad de Murcia, España. Tel.: +34 868884588. Correo: mferran@um.es

${ }^{2}$ Doctora por la Facultad de Educación. Profesora Titular de la Universidad de Murcia, España. Tel.: +34 868887782. Correo: carmenfg@um.es

${ }^{3}$ Doctora por la Facultad de Educación. Profesora Contratada Doctor de la Universidad de Murcia, España. Tel.: +34 868884587. Correo: m.gomez@um.es

${ }^{4}$ Doctora en Psicología. Profesora Catedrática de Universidad. Universidad de Murcia, España. Tel.: +34 868884072. Correo: lola@um.es

Revista Iberoamericana de Diagnóstico y Evaluación - e Avaliação Psicológica. RIDEP · No59 · Vol.2 · 35-47 · 2021

ISSN: 1135-3848 print /2183-6051online
} 


\section{Introducción}

La creatividad es un constructo importante tanto en psicología como en educación. Actualmente la definición más aceptada de creatividad entiende que ésta es la habilidad para crear algo que es a la vez original y valioso o útil (Runco \& Jaeg, 2012). La evaluación de la creatividad se fundamenta, principalmente, en las pruebas de pensamiento divergente. Entendiendo que este tipo de pensamiento es necesario aunque no suficiente por sí mismo para mostrar creatividad. De las pruebas utilizadas para medir la creatividad y el Pensamiento Divergente (PD), el más utilizado es el TTCT (Torrance, 1974). Tradicionalmente, son cuatro las dimensiones relacionadas con el PD: Fluidez (número de ideas que la persona es capaz de pensar); Flexibilidad (diferentes categorías, o enfoques utilizados); Originalidad (lo infrecuente y novedoso de la respuesta dada); y Elaboración (número de detalles no necesarios añadidos a la idea).

Hay un debate referido al procedimiento sobre cómo puntuar dichas dimensiones. Varios investigadores indican las principales controversias asociadas con la puntuación de las tareas de PD (Mouchiroud \& Lubart, 2001; Runco \& Acar, 2012; Runco \& Mraz, 1992; Runco, Okuda, \& Thurston, 1987). Primero, se cuestiona cómo se puntúa la dimensión de Originalidad o qué se debería considerar original. En el manual del TTCT, la puntuación normativa de Originalidad para cada respuesta está ligada a unos criterios de evaluación, diseñados hace tiempo; a pesar de las revisiones, dichos criterios se quedan obsoletos, porque no recogen nuevas respuestas. Además, el criterio para valorar la Originalidad no discrimina el tipo de respuestas según la edad del alumno.

Segundo, en el TTCT la Fluidez aparece como un "factor confluyente", porque las puntuaciones de Flexibilidad, Originalidad y Elaboración dependen mucho de número de respuestas, ya que se suma la puntuación obtenida en cada dibujo realizado; así pues, cuantas más respuestas ofrezca el estudiante, mayor será su puntuación [Un estudiante con una única respuesta muy original, puede obtener menor puntuación en el total que otro que presente muchas respuestas poco originales].
Es importante recordar que la puntuación total de Elaboración y Originalidad, obtenida sumando la puntuación de cada ítem (dibujo), ha sido utilizada en estudios previos que sugieren una estructura unidimensional del TTCT (Clapham, 1998; Clark \& Mirels, 1970; Kim 2006; Michael \& Wright, 1989; Runco, 1986; Seddon, 1983). Otros estudios han confirmado una estructura factorial donde las variables se agrupan por tareas $\mathrm{y}$ no por dimensiones, mostrando una alta correlación entre Fluidez, Flexibilidad y la Originalidad de las mismas tareas; lo que indica una mayor importancia del contenido de la tarea que de las dimensiones (Almeida, Prieto, Ferrando, Oliveira, \& Ferrándiz, 2008; Ferrando, Ferrándiz, Bermejo, Sánchez, Parra, \& Prieto, 2007; Prieto, López, Ferrándiz, \& Bermejo, 2003).

\section{Diferentes alternativas para puntuar la Originalidad}

La preocupación sobre si la Originalidad realmente depende de la Fluidez y qué relación existe entre ambas ha llevado a a estudiar cómo evitar la influencia de la puntuación en Fluidez sobre la de Originalidad para controlar la colinealidad entre ambas. Así, se han propuesto diferentes alternativas:

(1). Calcular la puntuación media (o promedio; o "ratio score") de la Originalidad. (Hocevar \& Michael, 1979; Runco \& Marz, 1992; Runco, Okuda, \& Thurston, 1991). (2). Considerar sólo la respuesta más original de las dadas. (Zarnegar, Hocevar, \& Michael, 1998). (3). Analizar sólo las tres primeras respuestas (Clark \& Mirels, 1970). (4). Solicitar al alumno que seleccione sus respuestas más originales (Michael \& Wright, 1989). (5). Puntuar sólo las respuestas únicas. (Runco, Okuda, \& Thurston, 1987). (6). Hay quienes proponen usar la siguiente fórmula: total de respuestas $=$ Fluidez + Originalidad (Hong \& Milgram, 1991; Moran, Milgran, Sawyer, \& Fu, 1983; Wallach \& Kogan, 1965). (7). Se ha propuesto otra ecuación en la cual la Fluidez $=$ el número de ideas distintas (Flexibilidad) + el número de repeticiones; estando la Flexibilidad implícita en la Fluidez, de forma que no constituyen indicadores distintos (Primi, Nakano, Morais, Almeida, \& David, 2013). (8). Recientemente, Silvia, Nusbaum y 
Beaty (2017) Sugieren clasificar las respuestas como originales o no, dependiendo de si las respuestas eran recordadas o inventadas durante la realización de la prueba.

Para evitar los problemas ligados a la puntuación de la Originalidad, otros proponen un método que excluye esa dimensión. Así, Snyder, Mitchell, Bossomaier y Pallier (2004) sugieren utilizar el Cociente Creativo, resultante de una función logarítmica en función de la Fluidez y la Flexibilidad. Mientras que Silvia, Martin y Nusbaum (2009) indican un 'snapshot scoring' (corrección instantánea), consistente en dar una puntuación holística a un conjunto de respuestas. Yendo más allá, Fürst y Grin (2018) proponen que los alumnos puntúen la creatividad de tres de sus compañeros.

De todos estos procedimientos, a nuestro modo de ver, el más fácil de utilizar por los profesionales de la Psicología y de la Educación sería la puntuación promedio, pues no requiere la aplicación de la prueba a un grupo de alumnos, ni el uso de análisis estadísticos, y tampoco requiere familiarizarse con nuevas rubricas de puntuación.

\section{Relación entre Fluidez y Originalidad}

Incluso considerando las alternativas en la puntuación de las dimensiones, sigue existiendo la duda de si la Originalidad podría realmente depender de la Fluidez del estudiante; es decir, si la correlación entre ambas variables no sea debida meramente a los procedimientos de puntuación, ni sea un artificio estadístico. Así, los individuos con mayor Originalidad serían también aquellos con mayor Fluidez (a los que se les ocurren muchas ideas).

En este sentido, Runco (1986) hizo un estudio utilizando la puntuación promedio de la Originalidad para dos tareas de PD (usos múltiples y significado de patrones) analizando la relación entre Originalidad y Fluidez de estas dos tareas. Para ello, diferenció entre los estudiantes que obtenían puntuaciones altas y bajas en creatividad y estudió si existían diferencias de rendimiento entre las respuestas de la primera y la segunda mitad de la tarea. Tanto para los estudiantes creativos como para sus compañeros las puntuaciones fueron más altas en la segunda mitad, aunque dichas diferencias no fueron estadísticamente significativas para los alumnos poco originales en la tarea de usos múltiples. Además, los estudiantes más originales siempre puntuaban más en Flexibilidad que sus compañeros, tanto para la primera como para la segunda mitad de la tarea.

Hong y Milgram (1991) utilizaron dos tipos de tareas para medir la creatividad: a) dos tareas estándar de creatividad en las que cualquier respuesta puede ser interpretada como válida (instancias y significado de patrones); y b) otra, más restrictiva, sobre resolución original de problemas. Las tareas se puntuaron diferenciando la Originalidad (respuestas que aparecían con menos del 5\% de frecuencia) de la Fluidez (se consideró como Fluidez aquellas respuestas populares que tenían más de un $5 \%$ de frecuencia). Estudiaron los resultados de los estudiantes muy originales versus los poco originales, en la primera y segunda mitad de la tarea. Encontrando que, para las medidas menos restrictivas, ambos tipos de estudiantes (los muy originales y los poco originales) mostraban un efecto significativo del orden; esto es, el número de respuestas originales se incrementaba, mientras que el número de respuestas frecuentes disminuía según iban respondiendo a las tareas.

Mouchiroud y Lubart (2001) analizaron si el método de puntuación que se maneje influye en la relación entre la Originalidad y la Fluidez, para ello, utilizaron cinco métodos de puntuación: 1) el propuesto en el manual del TTCT (sumando la puntuación de cada ítem); 2) las puntuaciones específicas para su muestra concreta; 3 ) el número de respuestas únicas; 4) la media de las ponderaciones; y 5) la mediana de las ponderaciones, correlacionaron cada puntuación de Originalidad con la Fluidez y Flexibilidad. Aunque la relación entre los índices de Fluidez y Originalidad fueron siempre estadísticamente significativos, éstos variaban en función del método de la puntuación de la Originalidad. Para el primer y el segundo método (puntuaciones sumativas de la Originalidad) se encontraron correlaciones más fuertes, mientras que utilizando los métodos tres, cuatro y cinco (puntuaciones no sumativas de la Originalidad), los datos mostraron correlaciones más débiles entre Originalidad y Fluidez, siendo las diferencias entre estas correlaciones estadísticamente significativas. 
Silvia et al. (2008) estudiaron si los diferentes índices de Originalidad (promedio, las dos mejores respuestas y la unicidad) estaban asociados al número de respuestas. Los datos indicaron que cuando la Originalidad se puntúa utilizando el promedio como índice y se usaban las dos mejores respuestas, se evitaba la influencia de la Fluidez sobre la Originalidad; mientras que cuando se utilizaba el índice de unicidad de respuestas, la correlación entre la Originalidad y la Fluidez era mayor. Es importante considerar que cuando las respuestas son ponderadas, según su frecuencia de aparición en una muestra dada, las muestras con mayor número de participantes están en desventaja, porque la probabilidad de tener una respuesta no repetida (única) decrece según aumenta el número de participantes (Mouchiroud \& Lubart, 2001).

Estos investigadores han contribuido a reconsiderar el sistema de puntuación y esto ha dado lugar a una información nueva y valiosa sobre la relación entre Fluidez y Originalidad. Aunque, han olvidado abordar uno de los problemas más importantes relacionados con la validez de constructo: si la estructura factorial de la prueba se ve afectada por el método de puntuación; es decir, si afecta a la diferenciación de las cuatro dimensiones clásicas del PD propuestas por Guilford (1962) y Torrance (1974). Esta cuestión es transcendental, especialmente porque muchos estudios no han encontrado la estructura por dimensiones (Fluidez, Flexibilidad, Originalidad y Elaboración) (Almeida et al., 2008; Clapham, 1998; Clark \& Mirels, 1970; Ferrando et al., 2007; Kim, 2006; Michael \& Wright, 1989; Prieto et al., 2006; Runco, 1986; Seddon, 1983).

\section{Objetivos}

Primero, examinar cómo afecta el procedimiento de puntuación en la validez de constructo del TTCT.

Antes de sugerir cualquier cambio sobre cómo puntuar el test, es importante saber si esto tendrá repercusiones reales en la práctica diaria educativa; por tanto, el segundo objetivo es estudiar si un cambio en el procedimiento de puntuación afectará en la identificación del potencial creativo. Tercero, examinar en qué medida las puntuaciones de Originalidad y Elaboración dependen de la Fluidez.

\section{Método}

\section{Participantes}

Han participado 649 estudiantes (319 chicos) de edades entre 5 y12 años $(M=8.26 ; D T=2.89)$ de diferentes escuelas públicas y concertadas con un estatus socio-económico medio pertenecientes a seis centros distintos en España. Se realizó un muestreo incidental no probabilístico. De ellos, el $15.7 \%$ estaban en la clase de Infantil 5 años, $14.2 \%$ en primero de Educación Primaria, $18.6 \%$ en segundo; $16.6 \%$ en tercero, $12.6 \%$ en cuarto; $9.7 \%$ en quinto y $12.6 \%$ en sexto de Educación Primaria.

\section{Instrumento}

Se ha utilizado el TTCT (Forma A Parte figurativa del Test de Pensamiento Creativo de Torrance. Se ha elegido la adaptación española de Prieto, López, Ferrándiz y Bermejo (2003). Compuesto por tres subtests: 1) hacer un dibujo (utilizando un papel verde) que valora la Originalidad y la Elaboración, 2) completar dibujos (formar dibujos a partir de unos trazos inacabados) y3) líneas paralelas (hacer dibujos utilizando líneas paralelas). Los subtest 2 y 3 valoran la Fluidez, la Flexibilidad, la Originalidad y la Elaboración.

Para cada uno de los subtest los alumnos tienen unos diez minutos de trabajo. La aplicación de la prueba requiere de unos 50 minutos aproximadamente.

Se ha escogido esta prueba porque permite aplicarla a niños con diferentes habilidades lectoescritoras, por lo que permite un amplio rango de edad en su aplicación.

Debido al tipo de ítems y al sistema de puntación de la prueba, ésta requiere no sólo de un índice de fiabilidad de la prueba como tal, sino también del estudio de la fiabilidad interjueces. Para este estudio, la fiabilidad de la prueba se midió utilizando el Alpha de Cronbach, introduciendo como elementos de la escala las puntuaciones de cada juez, los cuales fueron: .87 (Elaboración); .98 (Fluidez); .91 (Originalidad); y .97 (Flexibilidad). Y la fiabilidad interjueces se valoró utilizando índice de correlación intraclase, obteniendo valores adecuados (Anexo I). 


\section{Procedimiento}

Se contactó a distintos centros que mostraron interés en participar de esta investigación. Los directores informaron a los padres y pidieron las autorizaciones necesarias.

El test se administró en grupos, utilizando los tiempos de horario escolar. El test fue puntuado por tres jueces independientes formados en la evaluación del test, y se obtuvo una puntuación media de los tres.

El test se ha puntuado siguiendo las normas propuestas para la adaptación española (Prieto et al., 2003), de modo que la Fluidez se refiere al número de ideas (dibujos) que el estudiante aporta; la Flexibilidad o las diferentes categorías utilizadas; la Originalidad o nivel de infrecuencia (utilizando una rúbrica en la que las distintas respuestas han sido puntuadas según su novedad, facilitadas por Prieto, López, \& Ferrándiz, 2003); y la Elaboración o número de detalles no necesarios.

Se calcularon las puntuaciones sumativas totales para la Originalidad en cada subtest (sumando las puntuaciones de cada dibujo, según indica el manual del TTCT); y también se calculó el promedio total de la Originalidad (la puntuación sumativa dividida por el número de dibujos producidos: Fluidez). Lo mismo se hizo para la Elaboración, obteniendo una puntuación sumativa y otra promedio.

El análisis de datos se ha realizado con el programa estadístico SPSS V 24 para Windows (IBM, 2016).

\section{Análisis de datos}

A continuación, se detallan los análisis estadísticos realizados para acometer los objetivos de este estudio:

En primer lugar, previo al análisis de estructura interna del test de Torrance, las variables fueron sometidas a la prueba de normalidad establecida mediante la prueba de Kolmogorov-Smirnov. Se va a estudiar la linealidad de los datos utilizando los gráficos de nube de puntos. También se valoró la multicolinealidad utilizando el factor de inflación de la varianza (FIV), introduciendo como variable dependiente la Originalidad del subtest 1 y como independientes el resto de variables.

Además, para conocer la estructura interna y verificar la agrupación o independencia de las variables de la prueba se ha utilizado un análisis de componentes principales con rotación varimax. El uso de este análisis facilita la comparación con estudios previos, donde se utilizaron las puntuaciones sumativas de Originalidad y Elaboración (Ferrando et al., 2007). Para la realización de este análisis de componentes principales se utilizaron las puntuaciones promedio de la Originalidad y la Elaboración, y las puntuaciones sumativas de la Fluidez y la Flexibilidad. Se consideraron aquellos componentes con autovalores valor (eigenvalue) igual o superior a 1

Para comprobar si el cambio en la forma de puntuar la originalidad y la elaboración afectaría a la identificación de las personas consideradas creativas se realizó un análisis del índice de acuerdo de Kappa. Dicho índice indica el nivel de congruencia entre ambos procedimientos en cuanto a la clasificación de alumnos más y menos creativos. Para ello, se han agrupado a los alumnos en tres categorías: aquellos que obtienen altas puntuaciones ( $\mathrm{pc}>75$ ), bajas puntuaciones (pc <25), y puntuaciones medias (pc 25-75), siguiendo los criterios de Almeida y Freire (2008). Estos grupos se hicieron para las variables total Creatividad sumativa y Creatividad promedio; Originalidad sumativa y promedio, y la Elaboración sumativa y promedio.

Para estudiar la relación entre la fluidez con la originalidad y la elaboración se utilizaron dos procedimientos: a) el análisis de las correlaciones entre las variables y la comparación de dichas correlaciones y b) un estudio de los perfiles creativos de los estudiantes. Para ello, se clasificaron según su rendimiento en las distintas dimensiones (Fluidez, Originalidad y Elaboración), y se realizó una serie de análisis de varianza (ANOVAs) para verificar si los estudiantes más fluidos eran a su vez los más originales y/o elaborados y viceversa.

\section{Resultados}

Los resultados de normalidad establecieron la no normalidad de los datos. Dichos resultados pueden explicarse por el exceso de potencia estadística debido al gran tamaño muestral y la poca desviación de los datos. Como puede observarse 
Tabla 1. Estadísticos descriptivos de las variables del TTCT y Análisis Factorial Exploratorio de Componentes Principales utilizando las variables promedio de Originalidad y Elaboración

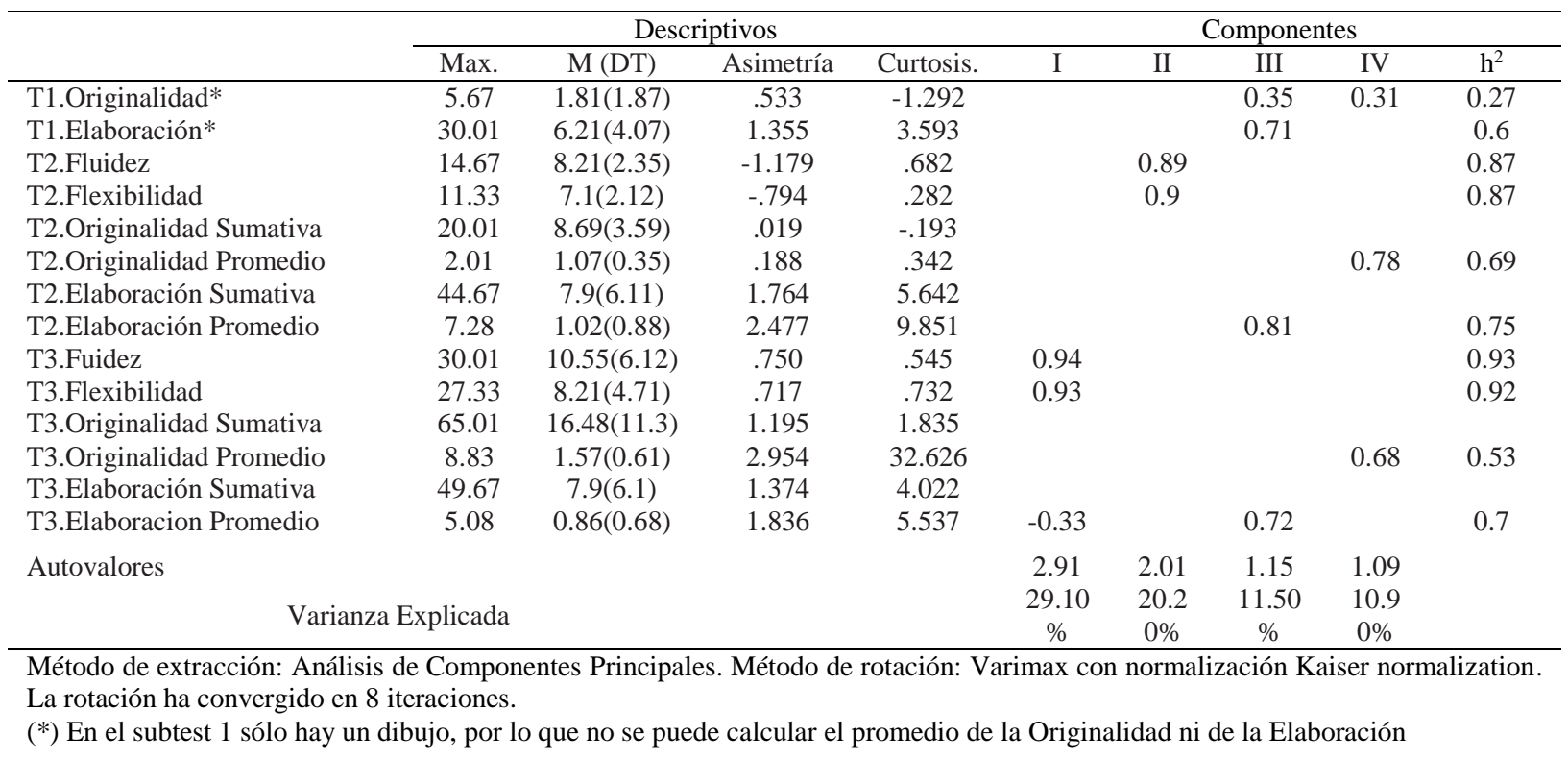

en el Anexo II los gráficos muestran una tendencia a la normalidad.

Respecto a la linealidad de los datos, la matriz de correlaciones mostró que un $22 \%$ de las correlaciones entre las variables tenían valores superiores a $r=.3$ (Anexo II). Respecto a la multicolinealidad, los resultados del diagnóstico de multicolinelidad estuvieron entre 1 y 7.4 , indicando que no hay síntomas de multicolinealidad.

Tras comprobar la factorialidad de los datos $\left[\mathrm{KMO}=.615\right.$, Barlett sphericity test: $\mathrm{X}^{2}=2634.631$; $\mathrm{df}=45, p<.001]$, los resultados del análisis de componentes principales (Tabla 1) indican que la Fluidez y la Flexibilidad no pueden asumirse como dimensiones independientes. Los componentes I y II muestran estas dos dimensiones entrelazadas. Estos resultados muestran la importancia de los requisitos/demandas de la tarea, más que de las habilidades creativas demandadas. Así, la Elaboración y la Originalidad (componentes III y IV, respectivamente) parecen ser más consistentes en términos de las habilidades creativas que demandan del estudiante, y no dependen tanto de los requisitos de la tarea. cuando usan las puntuaciones promedio de Originalidad y Elaboración en el análisis de componentes principales, éstas se desligan de la influencia de la Fluidez.

\section{¿Se siguen identificando a los mismos alumnos como creativos?}

La Figura 1 muestra las tablas de contingencia, gráficos de dispersión, coeficientes de correlación y el índice Kappa para las variables analizadas. Los índices de correlación son mayores para las variables sumativas y promedio de la Creatividad Total $(r=.954 ; p<.001)$, que para las de Elaboración $(r=.827 ; \quad p<.001), \quad$ y Originalidad $(r=.396 ; p<.001)$. El índice de acuerdo entre la puntuación sumativa y la promedio para la Creatividad Total fue alto (Kappa=.7202), lo que se refleja en el gráfico de dispersión, cuyos puntos siguen una línea recta. Para la Originalidad el nivel de acuerdo entre la puntuación sumativa y la promedio fue mucho más bajo (Kappa=.121), y el gráfico muestra una dispersión amplia de los casos (los puntos se concentran en las puntuaciones bajas y en las puntuaciones altas hay mayor desacuerdo). Para la Elaboración se alcanza un acuerdo moderado (Kappa $=.438)$.

\section{Relación de la Fluidez con Originalidad y Elaboración}

La correlación entre Originalidad y Fluidez fue significativamente mayor cuando se utilizan las puntuaciones sumativas de la Originalidad ( $r=.84$, $p<.001)$, que cuando se usaron las promedio ( $r=.09$, $p=.02) \quad[$ Steiger $\quad \mathrm{z}=25.28, \quad p<.001] . \quad$ Las correlaciones entre la Fluidez y la Elaboración 


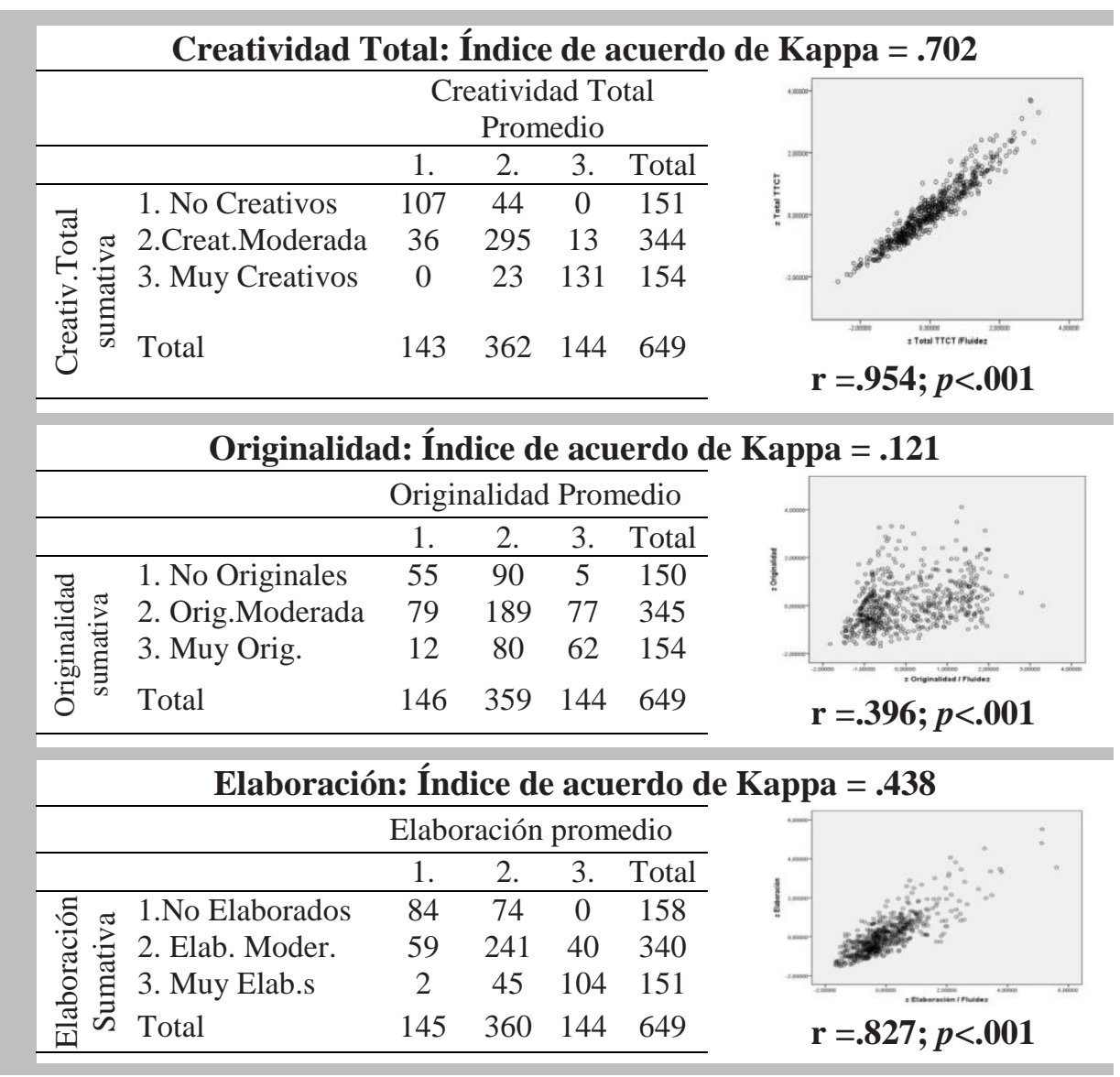

Figura 1. Índices de acuerdo, tablas de contingencia para las puntuaciones sumativas y promedio de las variables: Creatividad total, Originalidad y Elaboración

Tabla 2. Estadísticos descriptivos en Fluidez, Flexibilidad, Originalidad y Elaboración, F-MANOVA y análisis Post-hoc cuando se agrupa a los alumnos según su nivel de Fluidez, Originalidad promedio y

Elaboración Promedio

\begin{tabular}{|c|c|c|c|c|c|c|c|c|c|}
\hline & $\begin{array}{l}\text { 1. No } \\
\text { Fluidos }\end{array}$ & $\begin{array}{l}\text { 2. Fluidez } \\
\text { Moderada }\end{array}$ & $\begin{array}{l}\text { 3. Muy } \\
\text { Fluidos }\end{array}$ & $\begin{array}{c}\text { 4. No } \\
\text { Originales }\end{array}$ & $\begin{array}{l}\text { 5. Orig. } \\
\text { Moderada }\end{array}$ & $\begin{array}{c}6 \text { Muy } \\
\text { Originales }\end{array}$ & $\begin{array}{c}\text { 7. No } \\
\text { elaborados }\end{array}$ & $\begin{array}{l}\text { 8. Elab. } \\
\text { Modera. }\end{array}$ & $\begin{array}{c}\text { 9. Muy } \\
\text { elaborados }\end{array}$ \\
\hline Media de Fluidez & & & & 17.49 & 18.90 & 19.46 & 16.66 & 18.55 & 20.87 \\
\hline Desv.Típica de Fluidez & & & & 7.22 & 7.32 & 7.03 & 7.94 & 6.36 & 7.79 \\
\hline $\mathrm{N}$ alumnos & & & & 145 & 303 & 152 & 153 & 303 & 152 \\
\hline ANOVA y Post-hoc & & & & \multicolumn{3}{|c|}{$\begin{array}{c}F(2,597)=3 ; p=.050 ; \eta 2=.010 \\
{[4 \approx 5 ; 4<6 ; 5 \approx 6]}\end{array}$} & \multicolumn{3}{|c|}{$\begin{array}{c}F(2,605)=13.25 ; p<.001 \\
\eta 2=.042 ;[7<8 ; 7<9 ; 8<9]\end{array}$} \\
\hline Media de Flexibilidad & 8.72 & 15.31 & 22.16 & 13.79 & 15.44 & 16.48 & 13.36 & 15.32 & 17.11 \\
\hline Desv.Típica de Flexibilidad & 2.75 & 2.84 & 4.25 & 5.37 & 5.67 & 5.86 & 6.09 & 5.16 & 5.97 \\
\hline $\mathrm{N}$ alumnos & 158 & 301 & 149 & 145 & 303 & 152 & 153 & 303 & 152 \\
\hline ANOVA y Post-hoc & \multicolumn{3}{|c|}{$\begin{array}{l}F(2,602)=666.53 ; p<.001 \\
\eta 2=.688 ;[1<2 ; 1<3 ; 3>2]\end{array}$} & \multicolumn{3}{|c|}{$\begin{array}{c}F(2,597)=8550 ; p<.001 \\
\eta 2=.028 ;[4<5 ; 4<6 ; 5 \approx 6]\end{array}$} & \multicolumn{3}{|c|}{$\begin{array}{c}F(2,605)=16.99 ; p<.001 \\
\eta 2=.053 ;[7<8 ; 7<9 ; 8<9]\end{array}$} \\
\hline Media de Originalidad & 4.13 & 4.34 & 4.71 & & & & 3.77 & 4.52 & 4.71 \\
\hline Desv. Típica de Originalidad & 2.25 & 2.08 & 2.03 & & & & 2.11 & 2.06 & 2.12 \\
\hline $\mathrm{N}$ alumnos & 158 & 301 & 149 & & & & 153 & 303 & 152 \\
\hline ANOVA y Post-hoc & \multicolumn{3}{|c|}{$\begin{array}{c}F(2,605)=2.95 ; p=.53 ; \eta 2=.010 \\
{[1 \approx 2 ; 1 \approx 3 ; 3 \approx 2]}\end{array}$} & & & & \multicolumn{3}{|c|}{$\begin{array}{c}F(2,605)=9.14 ; p<.001 ; \eta 2=.29 \\
{[7<8 ; 7<9 ; 8 \approx 9]}\end{array}$} \\
\hline Media de Elaboración & 6.88 & 8.18 & 8.80 & 6.62 & 8.04 & 9.32 & & & \\
\hline Desv. Típica de Elaboración & 4.75 & 4.79 & 4.62 & 4.12 & 4.37 & 5.78 & & & \\
\hline $\mathrm{N}$ alumnos & 158 & 301 & 149 & 145 & 303 & 152 & & & \\
\hline ANOVA y Post-hoc & \multicolumn{3}{|c|}{$\begin{array}{c}F(2,605)=6.79 ; p=.001 ; \eta 2=.022 \\
{[1<2 ; 1<3 ; 3 \approx 2]}\end{array}$} & \multicolumn{3}{|c|}{$\begin{array}{l}F(2,597)=12.15 ; p<.001: \\
\eta 2=.039 ;[4<5 ; 4<6 ; 6>5]\end{array}$} & & & \\
\hline
\end{tabular}

sumativa $(r=.32, p<.001)$ y la Fluidez y la Elaboración promedio $(r=.16, \quad p<.001)$ no mostraron diferencias significativas [Steiger $\mathrm{z}=7.32, p=2.52]$.

La Tabla 2 muestra las puntuaciones medias de los distintos grupos de alumnos.
En primer lugar, cuando se agrupó a los estudiantes dependiendo de su nivel de Fluidez, los datos procedentes del análisis de varianza mostraron que hay un efecto de la Fluidez sobre las otras tres variables (Flexibilidad, Originalidad y Elaboración) [Wilk's lambda $=.041 ; F(3,603)=$ 4647.98; $\left.p<.001 ; \eta^{2}=.959\right]$. El efecto de la Fluidez 
fue estadísticamente significativo sobre la Elaboración promedio $[F(2,605)=6.79 ; p=.001$; $\left.\eta^{2}=.022\right]$ y sobre la Flexibilidad $[F(2,602)$ $\left.=666.53 ; p<.001 ; \eta^{2}=.688\right]$, pero no sobre la Originalidad $\left[F(2,605)=2.95 ; p=.53 ; \eta^{2}=.010\right]$.

En segundo lugar, cuando los estudiantes fueron agrupados según su nivel de Originalidad, los resultados no mostraron efectos significativos de la Originalidad promedio sobre la Elaboración, ni sobre la Fluidez ni sobre la Flexibilidad [Wilks's lambda $=.77 ; F(6,1192)=7.92 ; p<.001$, $\left.\eta^{2}=.038\right]$.

En tercer lugar, cuando los estudiantes se agruparon dependiendo de su nivel de Elaboración promedio, se halló un efecto significativo de la Elaboración sobre la Originalidad promedio, la Fluidez y la Flexibilidad [Wilks' lambda $=.925$ $\left.F(6,1206)=7.93 ; p<.001 ; \eta^{2}=.38\right]$. Estudiando los efectos post-hoc, las principales diferencias prevalecen cuando se comparan los grupos de baja Elaboración con los de alta y media Elaboración.

\section{Discusión y Conclusiones}

Este trabajo supone un intento por entender mejor la naturaleza del Pensamiento Divergente y la complejidad implicada en su evaluación cuando se utiliza el TTCT. Teniendo en cuenta la controversia en lo referente al impacto o influencia de los procedimientos de puntuación para la Originalidad y Elaboración, este estudio ha considerado el uso de métodos alternativos de puntuación con el fin de arrojar luz sobre la relación entre las cuatro variables clásicas del PD (Fluidez, Flexibilidad, Originalidad y Elaboración). Este método alternativo se basa en las puntuaciones promedio, que son consideradas como una medida válida más precisa y apropiada, que puede reducir el efecto de la Fluidez (Hocevar \& Michael, 1979; Runco \& Marz, 1992).

Respecto a la estructura interna del TTCT, nuestros resultados difieren de los estudios previos realizados en España y Portugal (Almeida et al., 2008; Ferrando et al., 2007; Oliveira et al., 2009). En dichos estudios se utilizaron las puntuaciones sumativas y se encontró que las variables del TTCT se agrupaban no por dimensiones, sino por subtests, dicho resultado se interpretó como que las demandas específicas de cada tarea pesaban más que las habilidades implicadas. Sin embargo, en nuestro estudio, al considerar las variables promedio de la Originalidad y la Elaboración, ambas dimensiones aparecen diferenciadas como factores independientes. Únicamente la Fluidez y Flexibilidad aparecen entrelazadas en los componentes I y II, lo que significa que están determinadas por las demandas de la tarea. En este caso, el resultado indica que la Fluidez y la Flexibilidad son fuertemente interdependientes.

Otro resultado relacionado con la estructura factorial de la prueba es que el componente de Originalidad (IV) es el último en emerger y el que menos varianza explica. Este resultado contradice la creencia de que el núcleo del Pensamiento Divergente/creativo radica en la Originalidad (Runco, 2008; Runco \& Jaeger, 2012), por tanto, requiere más investigación para clarificar la importancia de esta dimensión en el pensamiento creativo. Algunos niveles de distinción entre la Elaboración y la Originalidad habían sido verificados, y algunos autores habían sugerido que esto podría relacionarse con los enfoques creativos de adaptación-innovación propuestos por Kirton (Kim, 2006; Kim et al., 2006; Kirton, 1994).Este autor sugiere que hay dos perfiles de personas creativas: aquellas que prefieren adaptarse al medio y dan soluciones útiles, pero no rompedoras, y otro tipo de personas que se caracterizan por innovar y romper con las convenciones.

$\mathrm{Al}$ analizar si los cambios en la puntuación afecta a la identificación del potencial creativo, los datos indican que las personas consideradas creativas, lo son independientemente del método de puntuación (sumativo o promedio). Esto significa que en la práctica educativa cuando se identifican estudiantes superdotados, la puntuación promedio no afectaría a los puntos de corte para la nominación de estos estudiantes.

Finalmente, respecto a la relación entre Fluidez, Originalidad y Elaboración, se ha estudiado si las altas correlaciones encontradas en trabajos previos podrían deberse a un artificio estadístico. Para ello, se analizó si las mismas correlaciones se mantienen cuando se usan las puntuaciones sumativas y las promedio de la Originalidad y de la Elaboración. Utilizando las puntuaciones promedio, que reducen la influencia de la Fluidez sobre la Originalidad y la 
Elaboración, los coeficientes de correlación fueron más bajos, lo cual confirma los resultados de otros autores (Mouchiroud \& Lubart, 2001; Runco, 1986); sin embargo, la diferencia entre las correlaciones sólo fue estadísticamente significativa para la dimensión de Originalidad.

Además, los resultados del estudio de los perfiles creativos de los estudiantes dependiendo de su nivel de rendimiento en las distintas variables (Fluidez, Originalidad Promedio y Elaboración promedio), revelan lo siguiente:

a) Los estudiantes con mayor Fluidez no son necesariamente aquellos que muestran mayor Originalidad. Pero, los estudiantes altamente originales sí son los que manifiestan mayor Fluidez. Esto también ha sido corroborado por algunos expertos (Runco, 1986; Silvia et al. 2008). Las ideas inusuales ocurren cuando las convencionales ya han sido exploradas y no quedan más ideas comunes por explorar.

b) Los estudiantes con menor Fluidez son aquellos que también manifiestan menos Elaboración; mientras que los más elaborados son más fluidos. Este resultado contradice la asunción aceptada según la cual los límites de tiempo hacen que los alumnos elijan entre producir nuevas ideas o elaborar las que ya han dado. Nuestros resultados parecen indicar que la Fluidez es expresada no sólo en el número de dibujos, sino también en el número de detalles que se le añaden (en el futuro sería importante tener en consideración la relevancia de los detalles añadidos).

c) Los estudiantes más originales son también los más elaborados, y los menos originales son los que menos elaboran sus respuestas. Añadir detalles puede reflejar un conocimiento del objeto que se dibuja, pero también podría estar representando un insight (visión interna) en cuanto a qué detalles deberían añadirse.

Estos resultados apuntan a la existencia de una relación lineal entre las dimensiones o habilidades. Podría decirse que los individuos creativos normalmente presentan un perfil equilibrado ( $\sin$ picos), en lo que se refiere a las dimensiones de Fluidez, Originalidad y Elaboración. Así pues, cuando se usan las puntuaciones promedio, el PD es una combinación de las tres dimensiones (Fluidez, Originalidad y Elaboración), según lo que ya afirmaban los dos grandes pioneros (Guilford, 1962; Torrance, 1974).

Por último, algunas limitaciones de este estudio es que se ha probado sólo una de las posibilidades de puntuar la prueba. Además, en estudios futuros debería considerar si estos cambios en la puntuación afectan por igual a distintas pruebas de Pensamiento Divergente.

\section{Referencias}

Almeida, L., \& Freire, T. (2008). Metodologia da Investigação em Psicologia e Educação. Braga: Psiquilíbrios Edições

Almeida, L., Prieto, M. D., Ferrando, M., Oliveira, E., \& Ferrándiz, C. (2008). Torrance Test of Creative Thinking: The question of its construct validity. Thinking Skills and Creativity, 3(1), 53-58. doi:https://doi.org/10.1016/j.tsc.2008.03.003

Clapham, M. M. (1998). Structure of Figural Forms A and B of The Torrance Tests of Creative Thinking. Educational and Psychological Measurement, 58(2), 275-283. doi:https://doi.org/10.1177/0013164498058002 010

Clark, P. M., \& Mirels, H. L. (1970). Fluency as a pervasive element in the measurmemnt of creativity. Journal of Educational Measurement, 7(2), 83-86. doi:https://doi.org/10.1111/j.17453984.1970.tb00699.x

Ferrando, M., Ferrándiz, C., Bermejo, M. R., Sánchez, C., Parra, J., \& Prieto, M. D. (2007). Estructura interna y baremación del Test de Pensamiento Creativo de Torrance. Psicothema, 3(19), 489-496

Fürst, G., \& Grin, F. (2018). A comprehensive method for the measurement of everyday creativity. Thinking Skills and Creativity, 28, 84-97. doi:https://doi.org/10.1016/j.tsc.2018.03.007

Guilford, J. P. (1962). Factors that Aid and Hinder Creativity. Teachers College Record, 63, 380392.

Hocevar, D., \& Michael, W. B. (1979). The effects of scoring formulas on the discriminate validity of tests of divergent thinking. Educational and Psychological Measurement, 39(4), 917-921. 
doi:https://doi.org/10.1177/001316447903900 427

Hong, E., \& Milgram, R. M. (1991). Original thinking in preschool children: A validation of ideational fluency measures. Creativity Research Journal,4(3), 253-260. doi:https://doi.org/10.1080/10400419109534397

IBM Corp. Released 2016. IBM SPSS Statistics for Windows, Version 24.0. Armonk, NY: IBM Corp.

Kim, K. H. (2006). Is creativity unidimensional or multidimensional? Analyses of the Torrance Tests of Creative Thinking. Creativity Research Journal, 18(3), 251-259. doi:https://doi.org/10.1207/s15326934crj1803_2

Kim, K. H., Cramond, B., \& Bandalos, D. L. (2006). The latent structure and measurement invariance of scores on the Torrance Tests of Creative Thinking-Figural. Educational and Psychological Measurement, 66(3), 459-477. doi:https://doi.org/10.1177/0013164405282456

Kirton, M. J. (1994). Adaptors and innovators: Styles of creativity and problem solving. London: Routledge

Michael, W. B., \& Wright, C. R. (1989). Psychometric issues in the assessment of creativity. In J. A. Glover, R. R. Ronning, \& C. R. Reynolds (Eds.), Handbook of creativity (pp. 33-52). New York: Plenum Press. doi:https://doi.org/10.1007/978-1-4757-5356-1_2

Moran, J. D., Milgran, R. M., Sawyers, J., \& Fu, V. R. (1983). Original thinking in preschool children. Child Development, 54(4), 921-926. doi:https://doi.org/10.1111/j.14678624.1983.tb00513.x

Mouchiroud, C., \& Lubart, T. (2001) Childrens's original thinking: An empirical examination of alternative measures derived from divergent thinking task. Journal of Genetic Psychology, 162(4), 382-401. doi:https://doi.org/10.1080/00221320109597491

Oliveira, E. P., Almeida, L., Ferrándiz, C., Ferrando, M., Sáinz, M., \& Prieto, M. D. (2009). Tests de Pensamiento Creativo de Torrance (TTCT): Elementos para la validez del constructo en adolescentes portugueses. Psicothema, 21, 562-567.

Prieto, M. D., López, O., Ferrándiz, C., \& Bermejo, R. (2003). Adaptación de la prueba figurativa del Test de Pensamiento Creativo de Torrance en una muestra de alumnos de los primeros niveles educativos. Revista de Investigación Psicoeducativa, 21, 201-213.

Prieto, M. D., López Martínez, O., Ferrándiz García, C. (2003). La creatividad en el contexto escolar. Estrategias para favorecerla. Madrid: Pirámide.

Primi, R., Nakano, T. D. C., Morais, M. D. F., Almeida, L. S., \& David, A. P. M. (2013). Factorial structure analysis of the Torrance Test with Portuguese students. Estudos de Psicologia (Campinas), 30(1), 19-28. doi:https://doi.org/10.1590/s0103166x2013000100003

Runco, M. A. (1986). Flexibility and originality in children's divergent thinking. Journal of Psychology, 120(4), 345-352.

doi:https://doi.org/10.1080/00223980.1986.97 12632

Runco, M. A. (2008). Commentary: Divergent thinking is not synonymous with creativity. Psychology of Aesthetics, Creativity, and the Arts, 2(2), 93-96. doi:https://doi.org/10.1037/1931-3896.2.2.93

Runco, M. A., \& Acar, S. (2012). Divergent thinking as an indicator of creative potential. Creativity Research Journal, 24(1), 66-75. doi:https://doi.org/10.1080/10400419.2012.65 2929

Runco, M. A., \& Jaeger, G. J. (2012). The standard definition of creativity. Creativity Research Journal, 24(1), 92-96.

doi:https://doi.org/10.1080/10400419.2012.65 0092

Runco, M. A., \& Mraz, W. (1992). Scoring divergent thinking tests using total ideational output and a creativity index. Educational and Psychological measurement, 52, 213-221. doi:https://doi.org/10.1177/001316449205200 126

Runco, M. A., Okuda, S. M., \& Thurston, B. J. (1987). The psychometric properties of four systems for scoring divergent thinking tests. Journal of Psychoeducational Assessment, 5, 149-156.

doi:https://doi.org/10.1177/073428298700500 206

Runco, M. A., Okuda, S. M., \& Thurston, B. J. (1991). Environmental cues and divergent thinking. Divergent thinking, 79-85 
Seddon, G. M. (1983). The measurement and properties of divergent thinking ability as a single compound entity. Journal of Educational Measurement, 20(4), 393-402. doi:https://doi.org/10.1111/j.17453984.1983.tb00216.x

Silvia, P. J., Martin, C., \& Nusbaum, E. C. (2009). A snapshot of creativity: Evaluating a quick and simple method for assessing divergent thinking. Thinking Skills and Creativity, 4(2), 79-85.

doi:https://doi.org/10.1016/j.tsc.2009.06.005

Silvia, P. J., Nusbaum, E. C., \& Beaty, R. E. (2017). Old or new? Evaluating the old/new scoring method for divergent thinking tasks. The Journal of Creative Behavior, 51(3), 216-224. doi:https://doi.org/10.1002/jocb.101

Silvia, P. J., Winterstein, B. P., Willse, J. T., Barona, C. M., Cram, J. T., Hess, K. L., Martinez, L., \& Richard, C. A. (2008). Assessing creativity with divergent thinking tasks: Exploring the reliability and validity of new subjective scoring methods. Psychology of Aesthetics, Creativity and the Arts, 2(2), 68-85.

doi:https://doi.org/10.1037/1931-3896.2.2.68

Snyder, A., Mitchell, J., Bossomaier, T., \& Pallier, G. (2004). The creativity quotient: An objective scoring of ideational fluency. Creativity Research Journal, 16(4), 415-419. doi:https://doi.org/10.1080/10400410409534552

Torrance, E. P. (1974). The Torrance Tests of Creative Thinking - Norms-Technical Manual Research Edition - Verbal Tests, Forms $A$ and $B$ - Figural Tests, Forms $A$ and B. Princeton NJ: Personnel Press

Wallach, M. \& Kogan, N. (1965). Models of thinking in young children. New York: Holt, Rinehart \& Winston.

Zarnegar, Z., Hocevar, D., \& Michael, W. B. (1988) Components of original thinking in gifted children. Educational and Psychological Measurement, 48(1), 5-16. doi:https://doi.org/10.1177/001316448804800 103 


\section{Anexo I}

Fiabilidad del TTCT (índices de alfa de Cronbach y correlaciones intraclase para valorar la fiabilidad Inter jueces

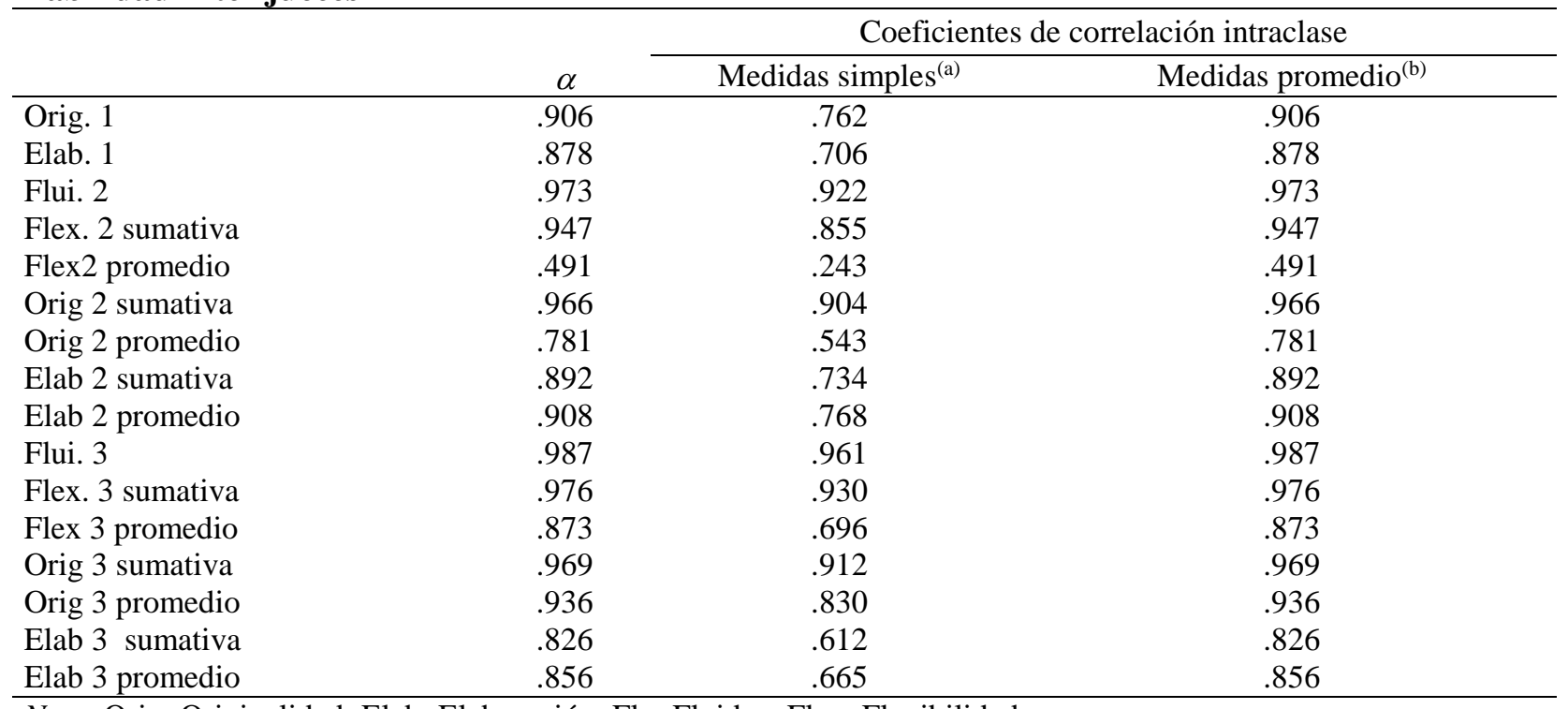

Nota. Orig: Originalidad; Elab: Elaboración; Flu: Fluidez; Flex: Flexibilidad

(a) El estimador es el mismo, esté presente o no el efecto de interacción

(b) Esta estimación se calcula asumiendo que el efecto de interacción está ausente, porque no es estimable de otra manera. 


\section{Anexo II}

\section{Gráficos de normalidad}

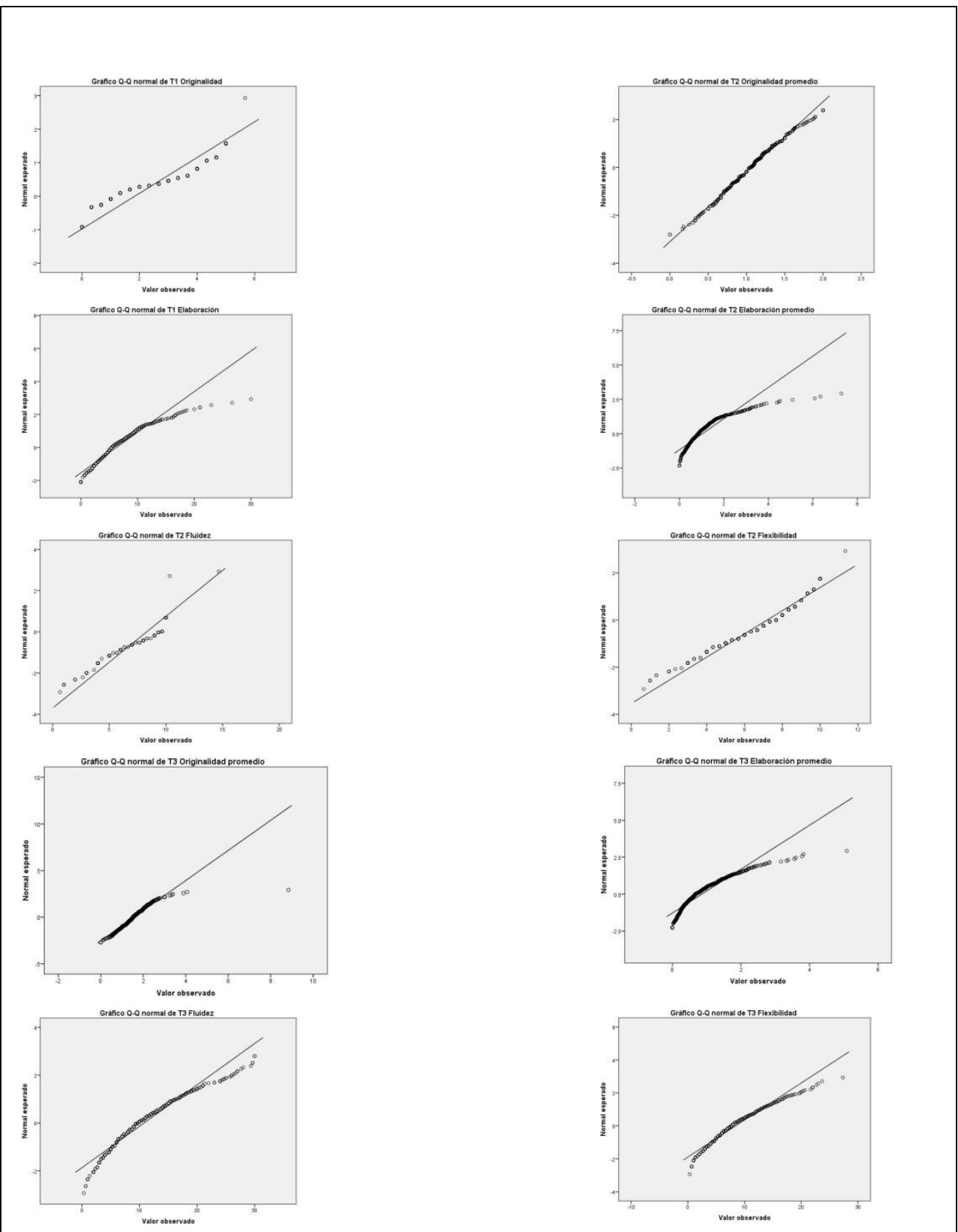

\section{Anexo III}

Matriz de correlaciones entre las variables sometidas al análisis de componentes principales

\begin{tabular}{|c|c|c|c|c|c|c|c|c|c|}
\hline & 1 & 2 & 3 & 4 & 5 & 6 & 7 & 8 & 9 \\
\hline 1. T1 Originalidad & 1 & & & & & & & & \\
\hline 2. T1 Elaboración & $.158^{* *}$ & 1 & & & & & & & \\
\hline 3. T2. Fluidez & .006 & $.084^{*}$ & 1 & & & & & & \\
\hline 4. T2. Flexibilidad & .043 & $.083^{*}$ & $.858^{* *}$ & 1 & & & & & \\
\hline 5. T2. Originalidad promedio & .063 & -.038 & -.066 & -.028 & 1 & & & & \\
\hline 6. T2 Elaboración promedio & $.137^{* * *}$ & $.409^{* *}$ & $-.258^{* *}$ & $-.236^{* *}$ & .038 & 1 & & & \\
\hline 7. T3 Flexibilidad & $.098^{*}$ & $.270^{* *}$ & $.370^{* *}$ & $.362^{* *}$ & .029 & $.081^{*}$ & 1 & & \\
\hline 8. T3. Fluidez & .068 & $.254^{* *}$ & $.392^{* *}$ & $.352^{* *}$ & .022 & .031 & .918 ** & 1 & \\
\hline 9. T3. Originalidad promedio & $.099^{*}$ & .078 & -.026 & -.001 & $.154^{* *}$ & $.138^{* *}$ & .073 & -.043 & 1 \\
\hline 10. T3 Elaboración promedio & $.102 *$ & $.242^{* * *}$ & $-.307^{* *}$ & $-.266^{* * *}$ & .063 & $.600^{* * *}$ & $-.236^{* *}$ & $-.305^{* *}$ & $.222^{* *}$ \\
\hline
\end{tabular}

